\title{
AVALIAÇÃO DE POTENCIAL PARA DESENVOLVER ALIMENTOS A PARTIR DE DESIDRATADOS OBTIDOS POR SPRAY-DRYING
}

\author{
T. RODRIGUES ${ }^{1 *}$, G. S. SILVA ${ }^{1}$, S. P. S. SANTOS ${ }^{1}$, A. R. S. TELES ${ }^{1}$, J. A. B. SANTOS ${ }^{1}$ G. F. \\ SILVA $^{2}$ \\ ${ }^{1}$ Universidade Federal de Sergipe, Departamento de Tecnologia de Alimentos \\ ${ }^{2}$ Universidade Federal de Sergipe, Laboratório de Tecnologias Alternativas \\ *e-mail: tassianarodrigues25@gmail.com
}

\begin{abstract}
RESUMO
Na cadeia produtiva de alimentos existem consideráveis perdas, desde a produção no campo até o momento de seu consumo in natura ou durante as etapas de industrialização, também gera quantidades elevadas de resíduos, sendo provenientes de matérias-primas que foram adquiridas algumas vezes a um alto preço, e que poderiam ser mais bem aproveitada na fabricação de produtos, ou transformadas em matéria-prima para serem comercializadas como insumos em outros processos. Este trabalho teve por objetivo a avaliação sensorial de produtos alimentícios desenvolvidos com desidratados de resíduos de abacaxi, acerola e maracujá obtidos por spraydrying na temperatura e concentrações de agente encapsulante: $20 \% / 120^{\circ} \mathrm{C}$ e $20 \% / 140^{\circ} \mathrm{C}$, $30 \% / 120^{\circ} \mathrm{C}$ e $30 \% / 140^{\circ} \mathrm{C}$. Foram feitas as analises físico-químicas ocorridas no processo a cada 30 dias, após a secagem, num período total de 90 dias, os resultados de todos os pós obtiveram perdas na sua propriedade ao longo do tempo, evidenciando-se que após os 60 dias ocorreram uma enorme degradação dos compostos fenólicos no pó de resíduos de acerola. Entretanto a umidade e atividade de água tiveram um aumento significativo, e a acidez desse pós houve um decréscimo no tempo de 30 dias. Com base na avaliação sensorial, verificou-se uma grande aceitação dos provadores com o produto produzido a partir do desidratado, onde 40\% "gostaram extremamente" e 63,34\% comprariam o produto.
\end{abstract}

\section{INTRODUÇÃO}

Há uma tendência mundial em relação ao mercado consumidor de frutas. É cada vez maior a demanda desses produtos devido ao seu valor nutricional (Oliva et al., 1996), e principalmente as frutas tropicais, pelo sabor exótico que possuem. $\mathrm{O}$ aumento do consumo de frutas em todo o mundo ocorre devido à procura dos consumidores por os novos sabores, atraídos por novos produtos. (MAIA et al, 2007).

Entretanto, existem consideráveis perdas na cadeia produtiva, desde a produção no campo até o momento de seu consumo in natura ou durante as etapas de industrialização, gerando quantidades elevadas de resíduos (MATSUURA, 2005; PEREIRA et.al.., 2006).

Além do desperdício, existe a crescente preocupação com seu descarte, uma vez que podem levar a problemas ambientais pela presença de substâncias de alto valor orgânico, para microrganismos, perdas de biomassa, energia, exigindo investimentos caros para o controle da poluição. (ABUD, 2009).

No entanto, o processamento de frutas pelas indústrias gera grandes quantidades de 
resíduos, que podem ser perfeitamente utilizados no desenvolvimento de novos produtos alimentícios, aumentando seu valor agregado. Seus descartes de resíduos, apresentam um grande problema quando são jogados de maneira incorreta sem tratamento prévio agravando a poluição ambiental (CEBDS, 2011). Portanto uma alternativa que vem sendo utilizada é a técnica de secagem por spray dryer, onde consiste na transformação de um produto no estado fluido para o estado sólido em forma de pó (LAOHASONGKRAM, et al., 2011).

Entretanto essa técnica preserva a qualidade nutricional, características como a aparência, o sabor e o odor dos mesmos. A aplicação tecnológica de subprodutos além de reduzir consideravelmente o resíduo desperdiçado, trás impacto positivo para economia (GIUNTINI et al., 2003). A utilização de novas tecnologias e o aperfeiçoamento das existentes, levando em conta a questão do desenvolvimento sustentável, encontra-se de forma intrínseca no aproveitamento consciente dos resíduos de alimentos para produção de subprodutos que serão integrados à alimentação humana.(FERRARI et al., 2004).

A produção de alimentos utilizando resíduos como matéria-prima caracteriza uma forma de aproveitamento desses resíduos, além de apresentar uma facilidade na forma de armazenamento e transporte, apresenta também maior facilidade de ser incrementada em outros alimentos, atuando assim como um coadjuvante alimentar de origem natural, de consumo direto ou a ser utilizado como ingrediente nas formulações de novos produtos.
O presente trabalho tem como principal objetivo avaliar $\mathrm{o}$ potencial para $\mathrm{o}$ desenvolvimento de novos produtos alimentícios, através do método de desidratação por spray dryer de resíduos de frutos tropicais, assim agregando valor comercial a um subproduto do processamento de alimentos, fazendo-o retornar à cadeia produtiva.

\section{MATERIAIS E MÉTODOS}

\subsection{ANÁLISES FÍSICO-QUÍMICAS}

A acidez titulável foi determinada seguindo a metodologia e realizando-se adaptações de acorde com o Instituto Adolfo Lutz, (2008).

Para atividade de água utilizou-se um higrômetro AQUALAB, modelo Series 3 TE, em triplicata.

O teor de Vitamina $\mathrm{C}$ foi determinado utilizando-se um método titulométrico, seguindo-se de adaptações. A solução de DCPIP foi padronizada com solução padrão de Ácido Ascórbico. A amostra foi macerada com solução de Ácido Oxálico, filtrada e completada para um balão; em seguida foi titulada com solução de DCPIP até viragem.

A determinação de densidade para amostras líquidas (resíduo aquoso) foi realizada seguindo a metodologia do Instituto Adolfo Lutz, (2008).

Para determinação de fenóis totais foi construída uma curva padrão de D-catequina em concentrações de $0,25,50,75,100,125$, 150 e $175 \mu \mathrm{g}$. A curva foi preparada utilizando-se $1 \mathrm{~mL}$ de cada concentração em tubos de ensaio distintos. Adicionou-se a cada tubo $5 \mathrm{~mL}$ de solução diluída de solução de 
Folin 10\%. Agitou em Vortex entre 30s e 8min, adicionou-se $4 \mathrm{~mL}$ de solução de carbonato de sódio $\left(\mathrm{Na}_{2} \mathrm{CO}_{3}\right) \quad 10 \%$ e agitou novamente em Vortex. Os tubos foram colocados por $1 \mathrm{~h}$ em banho termostático, a $30^{\circ} \mathrm{C}$, e depois transferidos para o banho de gelo por mais $1 \mathrm{~h}$. Em seguida, foi feita a leitura da absorbância em um espectrofotômetro a 700nm.

A umidade foi determinada em triplicata, pesando-se aproximadamente 2 gramas da amostra em cadinhos previamente tarados. (Instituto Adolfo Lutz, 2005).

$\mathrm{O} \mathrm{pH}$ foi determinado pelo processo potenciométrico, medindo em pHmetro e calibrando com soluções tamponadas de $\mathrm{pH}$ 4,0 e 7,0 (Instituto Adolfo Lutz, 2008).

$O$ teor de sólidos solúveis foi determinado por um refratômetro de bancada da marca ABBÉ (Instituto Adolfo Lutz, 2008).

\subsection{SECAGEM EM SPRAY DRYER}

Os resíduos das frutas tropicais foram processados em liquidificador com água destilada, na proporção 1:1 em massa, até obtenção de um suco. Em seguida foi adicionada enzima para realizar a quebra das fibras e esperou agir por alguns minutos e logo em seguida filtrou-se. A secagem foi realizada com as concentrações de agente encapsulante (maltodextrina) e temperatura, nas quais foram: $20 \% / 120^{\circ} \mathrm{C}$ e $20 \% / 140^{\circ} \mathrm{C}$, $30 \% / 120^{\circ} \mathrm{C}$ e $30 \% / 140^{\circ} \mathrm{C}$. O melhor resultado $\left(\left(20 \% / 120^{\circ} \mathrm{C}\right)\right.$ foi utilizado neste estudo.

\subsection{ANÁLISE SENSORIAL DE PRODUTOS}

A análise sensorial foi realizada com uma equipe de 30 julgadores não treinada e de idades variadas pertencentes à Universidade Federal de Sergipe. Foram oferecidos aos julgadores os produtos alimentícios desenvolvimentos com substituição parcial ou total de desidratados.

\section{RESULTADOS E DISCUSSÕES}

Na Tabela 1 e 2 estão apresentados os resultados obtidos das análises físicoquímicas do resíduo aquoso e dos desidratados. Os resultados das análises de ${ }^{\circ}$ Brix diferenciam-se bastante dos resíduos aquosos para os desidratados das frutas de abacaxi, maracujá e acerola. O teor de sólidos solúveis totais $\left({ }^{\circ}\right.$ Brix) é variável entre os frutos e teores de sólidos solúveis inferiores a $12{ }^{\circ}$ Brix são considerados imaturos (CQH/Ceagesp 2003).

Porém, as analises de atividade de água e umidade apresentaram um decréscimo em relação ao resíduo desidratado para o resíduo aquoso de abacaxi, maracujá e acerola, a umidade no resíduos aquosos fossem bem maiores, uma vez que o teor de água livre e muito elevado no fruto (CHAVES, 2004) e nos pó desidratados mostrou-se baixos teor de umidade, porém está no valor situado na faixa $5 \%$ a $10 \%$. dos teores de umidade para farinhas em pó especificadas pela (ANVISA, 2002) .

Nos resíduos das frutas ocorreu um aumento na acidez do resíduo aquoso para o desidratado, devido suas concentrações de ácidos cítrico e málico, da acidez total (Dull, 
1971). Os desidratado de acerola mostrou $\mathrm{pH}$ equivalência ao encontrado por Aquino et al, 2010 para a farinha, como muito ácido, pois apresenta valor de $\mathrm{pH}$ menor de 4,5, valor que delimita a proliferação microbiana (AQUINO, et al., 2010).

No parâmetro do teor de vitamina $\mathrm{C}$ para os resíduos aquosos e os resíduos desidratados não apresentou variação representativa nos resultado obtidos devido à degradação dos compostos e por ser facilmente degradável na presença de luz, em meio ácido, de oxigênio e de calor, entretanto o resíduo aquoso de acerola se encontra na faixa ótima de 1000-4500mg/100g (segundo LEUNG \& FOSTER, 1996), no qual foi de $1172,98 \mathrm{mg} / 100 \mathrm{~g}$ de vitamina C. Destacandose ainda o aumento de aproximadamente de 8 vezes no pó de acerola nos seus compostos fenólicos, devido à redução de água e ampliação das concentrações nos pós dos resíduos.

Tabela 1: Características físico-químicas dos resíduos aquosos de abacaxi, maracujá e acerola

\begin{tabular}{l|ccc}
\hline \multicolumn{1}{c|}{ Análises } & \multicolumn{3}{c}{ Res ultados dos resíduos aquosos das frutas } \\
\hline & ABACAXI & MARACUJÁ & ACEROLA \\
Acidez $(\mathrm{g} / 100 \mathrm{~g})$ & 3,05 & 5,38 & 19,70 \\
Atividade de Água & 0,998 & 0,992 & 0,304 \\
Vitamina C(mg/100g) & 19,66 & 7,40 & 1172,98 \\
$\mathrm{pH}$ & 3,88 & 4,5 & 3,27 \\
Teor Sólidos Solúveis(量Brix) & 6,6 & 4,8 & 4,08 \\
Fenóis $(\mu \mathrm{g} / \mathrm{g})$ & 0,80 & 0,30 & 3,60 \\
Densidade Aparente $(\mathrm{g} / \mathrm{mL})$ & 1,024 & 1,015 & 1,011 \\
Umidade $(\%)$ & 86,50 & 77,90 & 83,90 \\
\hline
\end{tabular}

Fonte: autor (2014)

Tabela 2: Características físico-químicas dos desidratados de abacaxi, maracujá e acerola

\begin{tabular}{l|ccc}
\hline \multicolumn{1}{c|}{ Análises } & \multicolumn{3}{c}{ Resultados dos desidratados de resíduos de frutas } \\
\hline & ABACAXI & MARACUJÁ & ACEROLA \\
Acidez $(\mathrm{g} / 100 \mathrm{~g})$ & 19,70 & 15,17 & 28,00 \\
Atividade de Água & 0,304 & 0,329 & 0,333 \\
Vitamina C(mg/100g) & 7,5 & 6,7 & - \\
pH & 3,35 & 4,48 & - \\
Teor Sólidos Solúveis( $\left({ }^{B}\right.$ riix) & 36,17 & 37,75 & 33,25 \\
Fenóis $(\mu \mathrm{g} / \mathrm{g})$ & 2,94 & 1,50 & 28,02 \\
Densidade Aparente $(\mathrm{g} / \mathrm{mL})$ & - & - & - \\
Umidade $(\%)$ & 4,02 & 4,60 & 3,37 \\
\hline
\end{tabular}

Fonte: autor (2014)
Depois da realização de secagem no Spray Dryer dos resíduos de frutas, os pós foram submetidos às mesmas análises físicoquímicas num período total de 90 dias, em intervalo de 30dias. Com o propósito de verificar à existência na alteração em suas propriedade físico-química, na Figura 1 podemos verificar os resultados de todos os pós e suas análises obtiveram diminuição nas sua propriedades com do tempo, destacandose uma enorme degradação dos compostos fenólicos no pó de resíduos de acerola após os 60 dias, porém nos outros desidratados teve uma pequena variação. A acidez dos pós desidratados houve um decréscimo ao decorrer de 30 dias e em seguida adquiriu-se uma elevação em sua porcentagem de acidez, a umidade e atividade de água ganhou um aumento significativo em sua variação.

Figura 1: Variação de Compostos Fenólicos, Teor de Umidade, Acidez e Atividade de água com o Tempo(T1 $=0$ dias, $\mathrm{T} 2=30$ dias, $\mathrm{T} 3=60$ dias, T4= 90 dias), entre os pós de Maracujá, Abacaxi e Acerola.

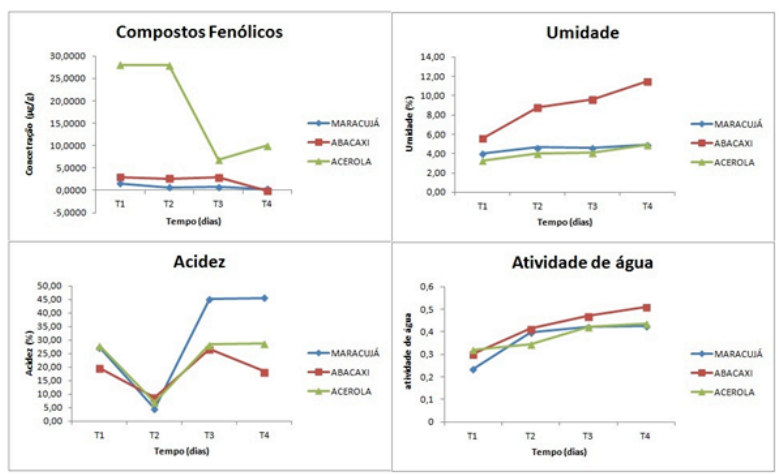

Fonte: autor (2014)

Como proposto no projeto, realizou-se a fabricação de um produto alimentício, onde o pó desidratado fosse incluso na mistura dos ingredientes para a fabricação do produto final. O produto feito foi um bolo, como 
mostra a Figura 3, com os respectivos ingredientes: farinha de trigo sem fermento, açúcar, ovos, leite, margarina, fermento e o pó do resíduo de abacaxi.

O bolo foi submetidos à análise sensorial para analisar se haveria aceitação do produto por parte dos degustadores. Avaliaram-se os parâmetros: Intenção de aceitação e de compra. Onde os parâmetros aceitação receberam nota de 1 (um) a 9 (nove), onde 1 (um) era "detestei extremamente" e 9 (nove) "gostei extremamente" e no parâmetro compra houve variação de 1 (um) a 5 (cinco), onde 1 (era) era "provavelmente não compraria" e 5 (cinco) " compraria certamente". Com base na avaliação, pode-se verificar que obteve-se um grande aceitação dos degustadores, como pode ser visto na Figura 2, no qual mais de $40 \%$ gostaram e comprariam o produto $(63,34 \%)$. Somente uma pessoa comentou sobre a textura do bolo, achou-o com aspecto bastante molhado.

Figura 2: Porcentagem de intenção de compra e aceitação dos degustadores.

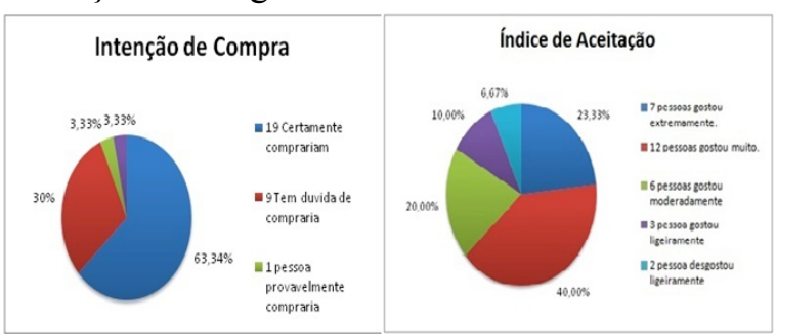

Fonte: autor (2014)

\section{CONCLUSÃO}

Em virtude dos resultados obtidos, verificamos que após serem submetidos à desidratação por spray-drying, percebeu-se a ocorrência de um aumento no valor de acidez, ${ }^{\circ}$ Brix e de compostos fenólicos em relação às amostras de resíduos aquoso. A atividade de água e umidade obteve uma diferença enorme, de modo que quando as amostras submetidas a spray-drying diminuem a quantidade de água livre nos resíduos.

$\mathrm{Na}$ analise sensorial realizada verificamos uma grande aceitação dos degustadores, sendo uma solução muito importante para a indústria, onde se tem enorme desperdício de resíduos, gerando vários problemas ambientais pela presença de substâncias de elevado valor orgânico. No entanto, esses resíduos podem aumentar seu valor, sendo perfeitamente utilizados no desenvolvimento de novos produtos alimentícios.

\section{REFERÊNCIAS}

ABUD, A. K. S. e NARAIN, N., "Incorporação da farinha de resíduo do processamento de polpa de fruta em biscoitos: uma alternativa de combate ao desperdício", BrazilianJournalofFood Technology, v. 12, n. 4, p. 257-265, 2009.

ANVISA. Farinhas. Resolução CNNPA no 12, Diário Oficial da União de 24 de julho de 1978. Disponível em: $<\mathrm{http}: / / w w w . a n v i s a . g o v \cdot b r /$ legis/resol/12_78_ farinhas.htm>. Acesso em: 1 jul. 2014.

AQUINO, A.C.M.S., LEÃO, K.M.M. Obtenção e caracterização físico-química da farinha de resíduos do processamento de polpa de acerola. Sergipe, 2009. Disponível em: $<$ www.hbatools.com.br/congresso/trabalho/42 /104050_1.doc $>$. Acessado em: 5 junho de 2014. 
CEBDS - Conselho Empresarial para o Desenvolvimento Sustentável, "A produção Mais Limpa na Micro e Pequena Empresa", Rio de Janeiro - RJ. Disponível em www.cebds.com. Acessado em junho de 2014.

CENTRO DE QUALIDADE DE HORTICULTURA (CQH)/COMPANHIA DE ENTREPOSTOS E ARMAZÉNS GERAIS DE SÃo PAULO (Ceagesp). Programa brasileiro para a modernização da horticultura: normas de classificação do abacaxi. São Paulo: Ceagesp, 2003. (Documentos, 24).

CHAVES, M. da C.V; GOUVEIA, J.P.G.; ALMEIDA, F. de A.C.; LEITE, J.C.A.; SILVA,

F.L.H. Caracterização físico-química do suco de acerola. Revista de Biologia e Ciências da Terra, v. 4, n. $2,2^{\circ}$ semestre 2004.

DULL, G.G. The pineapple: general. In: HULME, A.C. The biochemistry of fruits and their products. London: Academic Press, 1971. v.2, cap. 9A, p.303-324.

FERRARI, R.A., COLUSSI, F. e AYUB, R.A. "Caracterização de subprodutos da industrialização do maracujá Aproveitamento das sementes". Rev. Bras. Frutic., v. 26, n.1, 2004.
GIUNTINI, E. B., LAJOLO, F. M., MENEZES, E. W. Potencial de fibra alimentar em países ibero-americanos: alimentos, produtos e resíduos. Archivos Latinoamericanos de Nutrición, v.53, n.1, p.14-20, 2003.

INSTITUTO ADOLFO LUTZ (IAL). "Métodos físico-químicos para análise de alimentos". Coordenadores Odair Zenebon, NeusSadoccoPascuet e Paulo Tiglea - São Paulo: Instituto Adolfo Lutz, versão eletrônica, 2008.

LAOHASONGKRAM, K.; MAHAMAKTUDSANEE, $\mathrm{T}$.

$\&$ CHAIWANICHSIRI, S. Microencapsulation of Macadamia oil by spray drying.Procedia Food Science, n.1 p.1660-1665, 2011. common natural ingredients used in food drugs, and cosmetics 2ed. New York; Wiley, 1996. p6-7.

LEUNG， A.Y.;FOSTER， S. Acerola, In: Encyclopedia of common natural ingredients used in food, drugs, and cosmetic, 2ed. New York; Wiley, 1996.p 6-7. MAIA, G. A; SOUSA, P. H. M.; LIMA, A. S. Processamento de sucos de frutas tropicais. Fortaleza: UFC, 2007. 320 p. ( Shirley Janaine:Qualidade do Suco em Pó de misturas de frutas obtidos por Spray Drying).

MATSUURA, F.C.A.U. "Estudo do albedo de maracujá e de seu aproveitamento em barra de cereais". Tese (Doutorado em Engenharia de Alimentos) - Faculdade de Engenharia de Alimentos, Departamento de Tecnologia de Alimentos, Programa de PósGraduação em Engenharia de Alimentos, 
Universidade Estadual de Campinas,

Campinas, 2005. 